\title{
In cervical spondylotic myelopathy spinal cord motion is focally increased at the level of stenosis: a controlled cross-sectional study
}

\author{
Katharina Wolf $\mathbb{D}^{1,2} \cdot$ Markus Hupp $^{1} \cdot$ Susanne Friedl ${ }^{1} \cdot$ Reto Sutter $^{3} \cdot$ Markus Klarhöfer $^{4} \cdot$ Patrick Grabher $^{1}$. \\ Patrick Freund ${ }^{1,5,6,7} \cdot$ Armin $^{1, \text { Curt }^{1}}$
}

Received: 1 November 2017 / Revised: 22 January 2018 / Accepted: 1 February 2018 / Published online: 1 March 2018

(c) International Spinal Cord Society 2018

\begin{abstract}
Study design Level-, age-, and gender-matched controlled cross-sectional cohort study.

Objectives To investigate alterations of spinal cord (SC) motion within cervical spondylotic myelopathy (CSM) across the cervical spinal segments and its relation to cerebrospinal fluid (CSF)-flow, anatomic conditions, and clinical parameters.

Setting University Hospital Balgrist, Zurich, Switzerland.

Methods Overall, 12 patients suffering from CSM at level C5 and 12 controls underwent cardiac-gated 2D phase-contrastMRI at level C2 and C5 and standard MRI sequences. Parameters of interest: Velocity measurements of SC and CSF (area under the curve $=$ total displacement (normalization for duration of the heart cycle), total displacement ratio $(\mathrm{C} 5 / \mathrm{C} 2$; intraindividual normalization for confounders)), spinal canal diameters, clinical motor- and sensory scores, and performance measures.

Results Interrater reliability was excellent for SC motion at both levels and for CSF flow at C2, but not reliable for CSF flow at C5. Within controls, SC motion at C2 positively correlated with SC motion at C5 $(p=0.000)$; this correlation diminished in patients $(p=0.860)$. SC total displacement ratio was significantly increased in patients $(p=0.029)$ and correlated with clinical impairment $(p=0.017)$. Morphometric measures of the extent of stenosis were not related to SC motion or clinical symptoms. Conclusion The findings revealed physiological interactions of CSF flow and SC motion across the cervical spine in healthy controls while being diminished in CSM patients. Findings of focally increased SC motion at the level of stenosis were related to clinical impairment and might be promising as a diagnostic and prognostic marker in CSM.
\end{abstract}

Sponsorship CRPP Neurorehab of the University of Zurich, Switzerland.

\section{Introduction}

Phase-contrast MRI (PC-MRI) allows in vivo velocity measurements [1] using cardiac-gated data acquisition that

These authors contributed equally: Katharina Wolf, Markus Hupp.

Electronic supplementary material The online version of this article (https://doi.org/10.1038/s41393-018-0075-1) contains supplementary material, which is available to authorized users.

Katharina Wolf

katharina.wolf@uniklinik-freiburg.de

Spinal Cord Injury Center, Balgrist University Hospital, Zürich, Switzerland

2 Department of Neurology and Neurophysiology, University Hospital Freiburg, Freiburg, Germany

3 Department of Radiology, Balgrist University Hospital, can be mapped over the heart cycle resulting in a flowprofile. 2D PC-MRI assesses motion in one spatial direction per time. This non-invasive, dynamic-diagnostic approach enabled first pathophysiological insights within a variety of diseases presenting alterations in cerebrospinal fluid (CSF) dynamics [2]. Among patients with cervical spondylotic myelopathy (CSM), several studies applying 2D PC-MRI reported decreased CSF flow at level of stenosis [3, 4]. In addition, two groups focusing on spinal cord dynamics have described increased spinal cord motion at level of a cervical

Zürich, Switzerland

4 Siemens Healthcare AG, Zürich, Switzerland

5 Department of Brain Repair and Rehabilitation, Institute of Neurology, University College London, London, UK

6 Wellcome Trust Centre, University College London, London, UK

7 Department of Neurophysics, Max Planck Institute for Human Cognitive and Brain Sciences, Leipzig, Germany 
spinal stenosis [3, 5], related to sensory deficits in one study [3]. These studies were stimulated by the need of improving the diagnostic work-up in CSM beyond clinical and anatomical (i.e., static) MRI measures by evaluating dynamic factors contributing to the development of CSM [6]. A better insight into pathophysiological processes contributing to spinal cord dysfunction may be fundamental for understanding different disease courses that have been proven to be challenging when only relying on morphometric measures of stenosis. However, readily applicable clinical protocols using standard MRI scanners for 2D PC-MRI analysis of CSF flow and spinal cord motion have not yet been adopted for clinical diagnostic purposes. The relation of CSF- and spinal cord motion dynamics across the cervical spine and biometric factors (as age, sex, and body size) remains unclear [7, 8]. A strong effect of CSF dynamics within the cervical spinal canal due to venous outflow of the brain depending on breathing, central venous pressure, etc., has been demonstrated but is not yet quantified $[9,10]$. The applied velocity data implemented in former studies (e.g., means, maximum velocity, amplitudes) did not account for confounders. Therefor higher maximum velocities among patients might have been induced by manifold intraindividual biometric conditions (e.g., change of heart rates, blood pressure, morphology of cervical spinal segments) at time of investigation.

The aim of this study was to investigate the dynamics of spinal cord motion across the cervical spine and its relation to CSF dynamics in CSM patients in respect to individual biometric confounders as well as its correlation to clinical presentation. CSM patients with a stenosis at the cervical level C5, as the most common level of spinal canal stenosis [11], were investigated. We hypothesized that normalized dynamic spinal cord motion and CSF flow measures will reveal specific pathophysiological alterations in CSM patients, and these alterations may relate to the deterioration of spinal cord function.

\section{Methods}

\section{Study design}

This cross-sectional study was prospectively performed (2013-2015) and approved by the ethics committee (KEKZH 2012-0343). In total 12 patients and 12 age-matched and gender-matched healthy controls were enrolled, each group consisting of 7 males and 5 females. Inclusion criteria for patients were focal spinal canal narrowing with compression of the spinal cord at C5 and clinical symptoms consistent with CSM. Stenosis was defined as a loss of CSF signal in axial T2 MRI imaging. Clinical symptoms of CSM comprises pain, sensory or motor deterioration, gait problems, or bladder dysfunction. Any co-existing neurological disorder responsible for clinical symptoms was prospectively excluded by an independent neurologist. Controls were required to have no neurological symptoms nor relevant stenosis in MRI. Age for inclusion was $18-80$ years. All participants gave their written informed consent prior to study enrollment. Exclusion criteria consisted of general MRI-contraindications, epileptic seizures, mental illness, severe medical illness, and pregnancy. If controls showed an unexpected stenosis at level C2 or C5 in MRI as diagnosed by an independent and blinded radiologist they were considered as drop-outs.

All patients received a standardized clinical work-up assessing the mJOA score [12]. For more detailed information, motor and sensory scores of the upper limbs according to the International Standard for the Neurological Classification of Spinal Cord Injury (ISNCSCI) [13] were assessed. Motor assessment consisted of rating muscle strength of 5 upper extremity key muscles of each side according to the British Medical Research Council (MRC) strength grades from 0 to 5 resulting in total motor score with a maximum of 50 . Sensory assessment was conducted rating light touch and pin prick perception from 0 to $2(0=$ abolished perception, $1=$ impaired perception, $2=$ normal perception) in the 7 dermatomal key points $\mathrm{C} 2-\mathrm{T} 2$ for each side, resulting in a maximum total light touch (tot LT) and pin prick score (tot PP) of a maximum of 32 points, respectively. The Graded Redefined Assessment of Strengths, Sensibility and Prehension (GRASSP Version 1) [14] was performed as a detailed clinical assessment tool to measure the degree of upper limb impairment and dysfunction in the domains strength, sensation, and prehension. Therefore, testing key muscle strength, neurofilament sensory assessment, different grip types, and different tests for grasping function and fine motor skills (e.g., pouring water into a glass, screwing a nut, placing coins into slot) were performed. With the Spinal Cord Independence Measure (SCIM Version 3) [15], a functional assessment comprising of different subscores relating to body function in self-care (20 points), mobility (40 points) as well as bladder, bowel and breathing function (40 points) with a total score of 100 points was included. Healthy subjects all had mJOA score of 18 , were required to show full motor (50/50), sensory (32/32), and SCIM (100) scores and were assumed to show full GRASSP scores (232).

\section{Imaging parameters and analysis}

MRI-scans were performed at a 3T whole body MRI scanner (MAGNETOM Verio, Siemens Healthcare, Erlangen). Standard diagnostic T1w/T2w sequences were applied (T1 sagittal-Fov $220 \times 220 \mathrm{~mm}$, Matrix $288 \times 384$, TR $600 \mathrm{~ms}$, TE $10 \mathrm{~ms}$, slice thickness $3 \mathrm{~mm}$, averages 2, Flip 
angle $150^{\circ}$, Bandwidth $220 \mathrm{~Hz} / \mathrm{px}$; T2 axial-Fov $160 \times$ $160 \mathrm{~mm}$, Matrix $256 \times 320$, TR $3120 \mathrm{~ms}$, TE $93 \mathrm{~ms}$, slice thickness $3 \mathrm{~mm}$, averages 3 , Flip angle $150^{\circ}$, Bandwidth $285 \mathrm{~Hz} / \mathrm{px}$; T2 sagittal-Fov 220/220 mm, Matrix $288 \times$ 384, TR $3760 \mathrm{~ms}$, TE $87 \mathrm{~ms}$, slice thickness $2.5 \mathrm{~mm}$, averages 1, Flip angle $160^{\circ}$, Bandwidth $\left.260 \mathrm{~Hz} / \mathrm{px}\right)$. A retrospectively cardiac-gated commercially available phasecontrast sequence for craniocaudal velocity imaging was implemented (spatial resolution $0.5 \times 1.1 \times 5.0 \mathrm{~mm}^{3}$, FoV $140 \times 140 \mathrm{~mm}^{2}$, matrix $256 \times 128, \mathrm{TR}=11.5 \mathrm{~ms}, \mathrm{TE}=8.7$ ms, flip angle $10^{\circ}$, bandwidth $488 \mathrm{~Hz} / \mathrm{px}$ ). Velocity encoding of the sequence was set at $\pm 5 \mathrm{~cm} / \mathrm{s}$ for optimal CSF flow and spinal cord motion analysis in expectation of low CSF dynamics at level of stenosis as previously reported [3]. Phase-contrast MRI was acquired perpendicular to the spinal cord at level $\mathrm{C} 2$ and $\mathrm{C} 5$ consecutively with an acquisition time of $\sim 1.3 \mathrm{~min}$ per level depending on the heart rate. Flow velocities higher than the applied velocity encoding value $( \pm 5 \mathrm{~cm} / \mathrm{s})$ lead to inversed or "wrapped" velocity values. For correction of these values the program "ФUN" [16] was applied to regain true velocity values. An in-house Matlab script was used to analyze phase-contrast data. For each subject and level a standardized region of interest (ROI) was superimposed over the left and right CSF-space $\left(1.17 \mathrm{~mm}^{2}\right)$ that has been demonstrated to present highest CSF velocities [16, 17], and represents the assessable CSF-space within the comprised spinal canal. SC dynamics were analyzed by an into the spinal cord midcentered ROI $\left(19.68 \mathrm{~mm}^{2}\right)$. The averaged velocities of the voxels within the ROI were given at 20 time points within one cardiac cycle. Axial diameters of the spinal canal in ventro-dorsal (A) and left-to-right (B) directions were measured by clinically accustomed software tools in T2W imaging (AGFA IMPAX Viewing software).

\section{Data calculations and statistics}

The interrater reliability was examined by intraclass correlation coefficients (ICCs, single measures, two-way mixed effects model). The ICC values were characterized as follows: "fair" for 0.41-0.6, "moderate" for $0.61-0.8$, and "substantial" for $0.81-1$ [18]. ICC $\leq 0.6$ was not accepted for further analysis.

A deformity index (change from round to oval shape) of the spinal canal was calculated by division of ventro-dorsal over left-right diameter and expressed as "compression ratio" (A/B) [19]. Spinal cord motion was assessed by calculation of the area under the curve (AUC) using stepwise integration of the 20 velocity values $(\mathrm{cm} / \mathrm{s})$ over one heart cycle (s) per level. In case of negative velocities, the value was inversed before integration [6]. The AUC represents a normalization for the heart rate, which influences the duration of the diastole and therefore parameters like maximum velocity. The AUC illustrates the absolute motion $(\mathrm{mm})$ regardless to its cranial or caudal orientation over one heart cycle (SC total displacement; CSF total displacement). A ratio of SC total displacement between $\mathrm{C5}$ and $\mathrm{C} 2$ (SC total displacement ratio) per subject was implemented for intraindividual normalization in regard to intraindividual unknown biometric confounders (e.g., breathing, blood pressure, body height). Maximum (max.) velocities were assessed in regard to the analysis of intraindividual dynamics across the cervical spine but not used within interindividual comparisons (due to influence of the mentioned confounders). Statistical tests were performed by IBM SPSS software (Version 22). Values were reported as mean \pm standard deviation. For comparison of means the Student's $t$-test, for bivariate correlations the Pearson and Spearman- $\rho$ correlation coefficient was calculated and indicated as linear or monotone relationship respectively including age as confound of no interest. Significance was set to $p<0.05$.

\section{Results}

\section{Study characteristics}

The mean age was $46.05 \pm 10.05$ years in controls resp. $52.37 \pm 14.51$ years in patients $(p=0.228)$. Owing to the MRI-artifacts (1 case) and late notice of non-uniform acquisition of PC-MRI data at C5 (18 velocity measurements instead of 20 velocity measurements over one cardiac cycle; 3 patients), these incomplete MRI-datasets were excluded from analysis.

\section{Reliability}

The interrater reliability of all spinal cord motion measurements in controls and patients was excellent on both levels C2 and C5 (ICC > 0.8). CSF flow analysis at C2 was also reliable $(\mathrm{ICC}>0.8)$ but unreliable at $\mathrm{C5}(\mathrm{ICC}<0.4)$. Therefore, CSF analysis at C5 was excluded from further evaluation. ICC of each morphometric spinal canal assessment was acceptable at >0.61 (detailed ICCs see Table 1).

\section{Spinal cord motion and CSF flow-interactions across the cervical spine in physiological conditions and pathophysiological alterations in CSM}

There was no significant difference in spinal cord motion and CSF flow at the level C2 between controls and patients (Table 2). While in controls a strong trend for a positive correlation of CSF total displacement to SC total displacement at $\mathrm{C} 2$ could be observed, this relationship became significant in patients (Pearson's correlation coefficient $=$ 
Table 1 Summary of the intraclass correllation coefficients (two-way mixed model, single measures)

\begin{tabular}{llll}
\hline Level & Parameter & ICC & $p$-value \\
\hline C2 & Compression ratio & 0.63 & $4.10 \times 10^{-4}$ \\
& Anterior-dorsal spinal canal diameter & 0.83 & $1.76 \times 10^{-7}$ \\
& Max. CSF flow & 0.86 & $4.46 \times 10^{-8}$ \\
& AUC CSF flow & 0.99 & 0.00 \\
& Max. SC motion & 0.81 & $8.56 \times 10^{-7}$ \\
& AUC SC motion & 0.90 & $1.68 \times 10^{-9}$ \\
C5 & Compression ratio & 0.79 & $3.00 \times 10^{-6}$ \\
& Anterior-dorsal spinal canal diameter & 0.97 & $2.33 \times 10^{-15}$ \\
& Max. CSF flow & 0.35 & 0.07 \\
& AUC CSF flow & 0.35 & 0.06 \\
& Max. SC motion & 0.89 & $1.70 \times 10^{-8}$ \\
& AUC SC motion & 0.97 & $1.38 \times 10^{-13}$ \\
\hline
\end{tabular}

ICC intraclass correlation coefficient, $\max$. maximal, CSF cerebrospinal fluid, $A U C$ area under curve, $S C$ spinal cord

$0.857, p=0.001$ ) (Fig. 1), pointing to a preserved physiological relationship in patients at this level remote from stenosis.

In controls, there was no relevant increase of SC total displacement in craniocaudal direction ( $\mathrm{C} 2$ to $\mathrm{C} 5, p=$ 0.862) (Table 2). Accordingly, the SC total displacement ratio, was about $1(1.01 \pm 0.07)$, pointing to little difference between these segments. In patients, the SC total displacement ratio between $\mathrm{C} 2$ and $\mathrm{C} 5$ was significantly increased (controls: $1.01 \pm 0.07$, patients: $1.51 \pm 0.33 ; p=0.029$ ), indicating an increased SC motion at the focal compression site at level C5 (Fig. 2).

Relationships of CSF flow and SC motion at C2 and C5 observed in controls revealed interactions of spinal cord motion and CSF flow in physiological conditions across different segments (positive correlation of CSF maximum velocity at $\mathrm{C} 2$ to $\mathrm{SC}$ maximum velocity at $\mathrm{C} 5$ (Pearson's correlation coefficient $=0.728, p=0.011$ ); positive correlation of SC total displacement between C2 and C5 (Pearson's correlation coefficient $=0.953, p=0.000)$ ) was not evident in patients (Pearson's correlation coefficients $<0.5$, $p>0.5$ ) (Supplementary material: full dataset of all subjects see supplements, Table 3; video-examples of phase-contrast MRI of spinal cord motion at $\mathrm{C} 5$ of a control subject (video 1) and a patient (video 2)).

\section{Age and gender}

Within controls, but not within patients, age correlated positively with SC total displacement at C2 (Spearman's $\rho$ correlation coefficient $=0.618, p=0.043$ ). Gender did not correlate to any dynamic parameter.

\section{Spinal canal stenosis}

At level C2, there was no difference of spinal canal morphometry between controls and patients, whereas at C5 compression ratio and ventro-dorsal diameter were significantly decreased among patients $(p=0.000$, resp.). Among controls, CSF total displacement at $\mathrm{C} 2$ was negatively (linear) correlated with ventro-dorsal spinal canal diameter at C2 (Pearson's correlation coefficient $=-0.676$, $p=0.022$ ) and C5 (Pearson's correlation coefficient $=$ $-0.704, p=0.023)$. There was no correlation of spinal cord motion measures with spinal canal diameters or compression ratio among controls. Among patients, there was no correlation of spinal cord motion or CSF flow to spinal canal diameters or compression ratio.

\section{Clinical status}

A negative correlation of SC total displacement ratio to the light touch score (ISNCSCI) was observed among patients (Spearman's $\rho$ correlation coefficient $=-0.838, p=0.009$ ) (Fig. 3), and a trend towards negative relations to SCIM (Spearman's $\rho$ correlation coefficient $=-0.632, p=0.09$ ). There was no other significant relation among other clinical parameters (Pearson's correlation coefficient $< \pm 0.5$ ).

\section{Discussion}

The present study focused on physiological relations of CSF flow and spinal cord motion across the cervical spine in healthy controls and pathophysiological changes in CSM patients. The interaction of CSF flow and SCM is rather unknown and has not yet sufficiently distinguished between systemic and focal dynamic changes across different spinal levels in CSM.

Interrater reliability of the dynamic measurements was excellent for spinal cord motion across all cervical segments while measures of CSF flow were less reliable at C5 level. This might be mostly attributed to small ROIs in CSF measurements according to minor remaining subarachanoidal space and inhomogeneous CSF flow compared to solid spinal cord motion measurements. In viscous fluids, higher velocities occur in the center of the fluid due to traction effects at its boundaries [3]. Minor asynchronous multidirectional flows within the upper cervical spine have been shown in healthy controls [17]. Bunck et al. [20] described anterolateral jets and turbulences at the level of spinal canal compression in one case using 4D PC-MRI supporting the presumption of complex CSF dynamics at level of stenosis and the limitations of 2D PC-MRI by only assessing craniocaudal motion. Also, poorer MRI signal to noise ratio in lower cervical segments have to be taken into 
Table 2 CSF and spinal cord (SC) total displacement (area under the curve)

\begin{tabular}{lll}
\hline & \multicolumn{2}{l}{ Mean \pm standard deviation } \\
\cline { 3 - 3 } & Controls & Patients \\
\hline CSF and spinal cord $(S C)$ total displacement (= area \\
under the curve)
\end{tabular}

$S C$ spinal cord, CSF cerebrospinal fluid, max. maximal

*Significant value

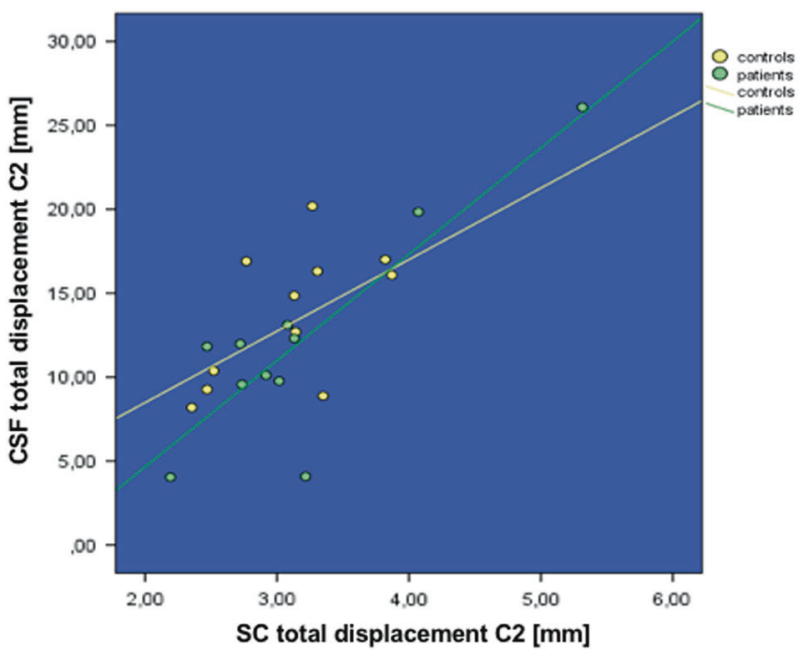

Fig. 1 In patients there is a positive (linear) relation of CSF total displacement at $\mathrm{C} 2$ to total spinal cord (SC) total displacement at $\mathrm{C} 2$; among controls there is a strong trend (controls: Pearson's correlation coefficient $=0.598, p=0.052$; patients: Pearson's correlation coefficient $=0.857, p=0.001$ )

account, whereas this did not influence spinal cord motion reliability at $\mathrm{C} 5$ in our study. In contrast to the complex dynamic properties of the CSF, the motion of the spinal cord as a solid morphological structure could be shown to be reliably assessable.

Physiological CSF flow within the spinal canal derives mostly from external pressure gradients [21]. Therefore different influence factors as possible confounders have to be taken into account in measuring CSF flow and spinal cord motion. In normal breathing conditions, CSF shifts towards the brain during the diastolic phase and inspiration resulting in an upward CSF flow within the cervical spine [22]. Recent 2D PC-MRI-studies confirmed, that deep abdominal respiration results in highest CSF velocities in

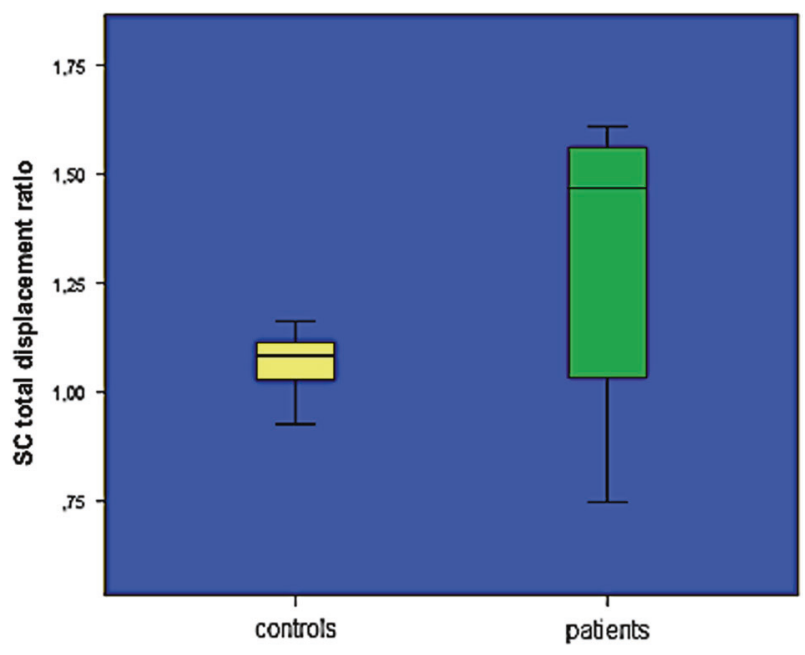

Fig. 2 Spinal cord (SC) total displacement ratio: within controls, the relation of the spinal cord total displacement between $\mathrm{C} 5$ and $\mathrm{C} 2$ is close to one pointing to undisturbed movement dynamics between these segments. Within patients, the spinal cord total displacement ratio is significantly altered, showing a specifically local increase in spinal cord motion at the stenotic level $(p=0.029)$

cranial direction $[9,10]$ while increased heart rates result in shorter diastolic phases and therefore decreased CSF flow [2]. A physiological increase of CSF velocities in craniocaudal direction across the cervical spinal levels has been noticed before [17], with a maximum at level C5 (revealed by 4D PC-MRI-techniques (3 spatial directions $\times$ time) [20]. This might be attributed to the decrease of subarachnoidal space according to the law of Hagen-Poiseuille.

Physiological, pulse-synchronic oscillations of the spinal cord have been observed and described in prior studies [23-25]. In physiological conditions, the origin of spinal cord motion was diversely attributed, mostly to remote factors like intracranial CNS- and CSF-pulsation within the cardiac cycle [23, 25] and breathing [26]. 


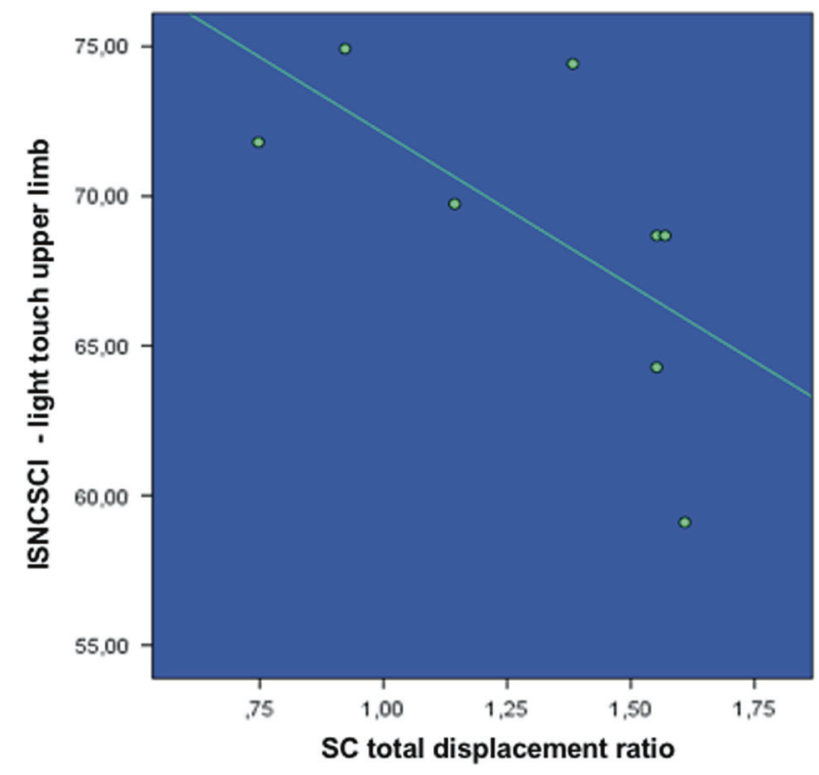

Fig. $3 \mathrm{CSM}$ patients show a negative (monotone) relation of spinal cord (SC) total displacement ratio (ratio of area under the curve (AUC) at $\mathrm{C} 5$ to $\mathrm{AUC}$ at $\mathrm{C} 2$ ) to clinical presentation (ISNCSCI-light touch upper limb); Spearman's $\rho$ correlation coefficient $=-0.802, p=0.017$

Interestingly, also focal influences at the spinal segments due to expansion of segmental arteries (oscillations became diminished after transection of the local vascular support (canine-model) [24] and due to local CSF flow alterations were discussed to influence spinal cord motion [11, 27-29].

As spinal cord motion and CSF flow can be influenced by many individual confounders (e.g., breathing, blood pressure, heart rate), a correction for these factors seems to be required for comparison between subjects. The AUC accounts for the duration of the heart cycle, as it was shown that shorter diastolic phases cause decreased CSF flow [2]. For comparison between different subjects, also different unknown and varying confounders have to be taken into account. Therefore, we introduced a normalization method calculating a ratio for SC motion with the reference level $\mathrm{C} 2$. This segment is very seldom affected by cervical stenosis, and confounders are likely to influence both segments in the same amount within the same subject. The implemented SC total displacement ratio is therefore the most valuable parameter within the presented results. Uncorrected absolute velocity values (e.g., maximum velocity, amplitude of velocity signal, mean velocity) might falsely indicate relevant findings.

Among controls, a negative correlation between CSF total displacement and the size of the spinal canal (ventrodorsal diameter) follows the law of Hagen-Poiseuille and is in accordance to a previous report [20]. A positive relation of age and spinal cord motion among controls was confirmed [30], but could not be shown in this patient population, possibly due to small patient numbers.

Within our results, a physiological relationship between spinal cord motion across different segments respectively between CSF flow and spinal cord motion could be observed in controls, and in patients at the non-affected segment C2. In controls, the observed trend of a slight increase of spinal cord motion towards $\mathrm{C} 5$ might underline the close interaction of CSF flow and spinal cord motion as an increase of CSF flow across the cervical spine towards C5 was previously reported [20]. While physiological correlations were preserved at $\mathrm{C} 2$ level in patients, they diminished at the stenotic level C5, emphasizing focal dynamic changes at the level of stenosis. Our results demonstrated a specifically focal increase of spinal cord motion at the level of stenosis in CSM patients but preserved dynamics rostral the stenotic segment.

Among CSM patients, our results indicated a pathophysiological imbalance of the dynamic connectivity across the cervical spine most likely due to the locally decreased subarachnoidal space. Analog to the theory of focal dynamic influences at the segmental levels [29], it could be hypothesized, that due to decreased subarachnoidal space, local pressures can no longer be compensated by CSF and therefore are transduced directly to spinal cord tissue resulting in increased motion and therefor elevated mechanical stress beyond compression due to anatomical stenosis. Elevated dynamic stress is therefore supposed to subsequently lead to deterioration of spinal cord function.

The focal pathologic increase of spinal cord motion at the level of stenosis in CSM patients was also related to clinical impairment. A correlation of increased SC displacement ratio to sensory deficits and a trend towards functional impairment could be observed [22]. Therefore, elevated mechanical stress due to increased spinal cord motion is supposed to lead to deterioration of the spinal cord.

Measuring spinal cord motion as a correlate of dynamic stress onto spinal cord tissue might serve as parameter to monitor the further disease course within future studies. Excellent reliability of spinal cord motion measurements by 2D phase-contrast MRI underlines the value for further clinical studies. As axial phase-contrast MRI sequences last only about $1.3 \mathrm{~min}$ per level, it is implementable without any difficulty into clinical protocols.

This study had some limitations. The small number of subjects and unfortunately some incomplete datasets. Despite small numbers, results were significant. Owing to these small group sizes, further, large prospective studies are needed towards more conclusive insights of normal values and towards a more profound understanding of the pathomechanisms. 


\section{Conclusion}

This is the first study to show that increased spinal cord motion occurs specifically at the level of stenosis while dynamics at level C2 rostral from stenotic segments remain physiological in CSM patients. Increased spinal cord motion relates to clinical impairment revealing a relevant impact on spinal cord integrity in CSM patients. Spinal cord motion can be easily assessed in routine protocols (2D PCMRI) with minor additional scanning time (about $1.3 \mathrm{~min}$ per level) and excellent reliability. Normalization procedures for different confounders were introduced for the first time. Therefore, measures of spinal cord motion may be considered in diagnostic work-up to reveal the impact of CSM on spinal cord function complementing measures of spinal canal stenosis.

Acknowledgements We would like to thank PD Dr. Martin Schubert and the staff of the Neurophysiology Lab, Spinal Cord Injury Center, Balgrist University Hospital Zürich, Switzerland and Alexandra Naef and Barbara Wagner, Department of Radiology, University Hospital Balgrist, Zürich, Switzerland.

Funding The study was supported by the CRPP Neurorehab of the University of Zurich, Switzerland. The study was approved by the ethics committee of the University of Zurich, KEK-ZH 2012-0343; written informed consent off all participants for publication was received.

\section{Compliance with ethical standards}

Conflict of interest MK is employed by Siemens Healthcare AG, Zürich, Switzerland. The remaining authors declare that they have no conflict of interest.

\section{References}

1. Yamada A, Tsuchiya K, Bradley WG, Law M, Winkler ML, Borzage MR, et al. Current and emerging MR imaging techniques for the diagnosis ad management of CSF flow disorders: a review of phase-contrast and time-spatial labeling inversion pulse. Am J Neuroradiol. 2015;36:623-30.

2. Bradley WG, Haughton V, Mardal KA. Cerebrospinal fluid flow in adults. Hand Clin Neurol. 2016;135:591-601.

3. Vavasour IM, Meyers SM, MacMillan EL, Mädler B, Li DK, Rauscher A, et al. Increased spinal cord movements in cervical spondylotic myelopathy. Spine J. 2013;14:2344-54.

4. Shibuya R, Yonenobu K, Koizumi T, Kato Y, Mitta M, Yoshikawa H. Pulsatile cerebrospinal fluid flow measurement using phase-contrast magnetic resonance imaging in patients with cervical myelopathy. Spine. 2002;27:1087-93.

5. Chang HS, Nejo T, Yoshida S, Oya S, Matsui T. Increased flow signal in compressed segments of the spinal cord in patients with cervical spondylotoc myelopathy. Spine. 2014;39:2136-42.

6. Kalsi-Ryan S, Karadimas SK, Fehlings MG. Cervical spondylotic myelopathy: the clinical phenomenon and the current pathobiology of an increasingly prevalent and devastating disorder. Neuroscientist. 2013;19:409-21.

7. Schmid Daners M, Knoblich V, Soellinger M, Boesinger P, Seifert B, Guzzella L, et al. Age-specific characteristic and coupling of cerebral arterial inflow and cerebrospinal fluid dynamics. PLoS ONE. 2012;7:e37502.

8. Oner Z, Kahraman AS, Kose AO, Kavakli A, Cay M, Ozbag D. Quantitative evaluation of normal aqueductal cerebrospinal fluid flow using phase-contrast cine MRI according to age and sex. Anat Rec. 2017;300:549-55.

9. Dreha-Kulaczewski S, Joseph AA, Merboldt KD, Ludwig HC, Gärtner J, Frahm J. Identification of the upward movement of human cerebrospinal fluid in vivo and its relation to the brain venous system. J Neurosci. 2017;37:2395-402.

10. Yildiz S, Thyagaraj S, Jin N, Zhong X, Plavian SH, Marin BA, et al. Quantifying the influence of respiration and cardiac pulsations on cerebrospoinal fluid dynamics using real-time phasecontrast MRI. J Magn Reson Imaging. 2017;46:431-9.

11. Baron EM, Young WF. Cervical spondylotic myelopathy: a brief review of its pathophysiology, clinical course, and diagnosis. Neurosurgery. 2007;60:35-41.

12. Kato S, Shima A, Oka H, Chikuda H, Takeshida Y, Miyoshi K, et al. Comparison of the Japanese Orthopaedic Association (JOA) Score and Modified JOA (mJOA) Score for the assessment of cervical myelopathy: a multi-center observational study. PLoS ONE. 2015;10:e123022. https://doi.org/10.1371/journal.pone. 0123022 .

13. Kirshblum SF, Waring W, Biering-Sorensen F, Burns SP, Johansen M, Schmidt-Read M, et al. Reference for the 2011 revision of the International Standard for Neurological Classification of Spinal Cord Injury. J Spinal Cord Med. 2011;34:547-54.

14. Kalsi-Ryan S, Curt A, Verrier MC, Fehlings MG. Development of the graded redefined assessment of strength, sensibility and prehension (GRASSP): reviewing measurement specific to the upper limb in tetraplegia. J Neurosurg Spine. 2012;17:65-76.

15. Itzkovich M, Gelernter I, Biering-Sorensen F, Weeks C, Laramee MT, Craven BC, et al. The spinal cord independence measure (SCIM) version III: reliability and validity in a multi-center international study. Disabil Rehabil. 2007;29:1926-33.

16. Witoszynskyj S, Rauscher A, Reichenbach JR, Barth M. Phase unwrapping of MR images using Phi UN-a fast and robust region growing algorith. Med Image Anal. 2009;13:257-68.

17. Haughton V, Mardal KA. Spinal fluid biomechanics and imaging: an update for neuropradiologists. Am $\mathrm{J}$ Neuroradiol. 2014;35:1864-9.

18. Shrout PE, Fleiss JL. Intraclass correlations: uses in assessing intra rater reliability. Psych Bull. 1979;86:420-38.

19. Shin JJ, Jin BH, Kim KS, Cho YE, Xho WH. Intramedullary high signal intensity and neurological status as prognostic factors in cervical spondylotic myelopathy. Acta Neurochir. 2010;152:1678-94.

20. Bunck AC, Kroger JP, Juttner A, Brentrup A, Fiedler B, Schaarschmidt F, et al. Magnetic resonance 4D flow characteristics of cerebrospinal fluid at the craniocervical junction and the cervical spinal canal. Eur Radiol. 2011;21:1788-1796. 29

21. Yamada S, Kelly E. Cerebrospinal fluid dynamics and the pathophysiology of hydrocephalus: new concepts. Semin Ultrasound CT MR. 2016;37:84-91.

22. Feinberg DA, Mark AS. Human brain motion and cerebrospinal fluid circulation demonstrated with MR velocity imaging. Radiology. 1987;163:793-9.

23. Enzmann DR, Pelc NJ. Brain motion: measurement with phasecontrast MR imaging. Radiology. 1992;185:653-60.

24. Matsuzaki H, Wakabayashi $\mathrm{K}$, Ishihara $\mathrm{K}$, Ishikawa $\mathrm{H}$, Kawabata $\mathrm{H}$, Onomura $\mathrm{T}$. The origin and significance of spinal cord pulsation. Spinal Cord. 1996;34:422-8.

25. Tanaka H, Sakurai K, Kashiwagi N, Fujita N, Hirabuki N, INaba $\mathrm{F}$, et al. Transition of the craniocaudal velocity of the spinal cord: from cervical segment to lumbar enlargement. Invest Radiol. 1998;33:141-5. 
26. Winklhofer S, Schroth F, Stolzmann P, Krings T, Mull M, Wiesmann M, et al. Spinal cord motion: influence of respiration and cardiac cycle. Rofo. 2014;136:1016-21.

27. Schaller B, Graf R. Different compartments of intracranial pressure and its relationship to cerebral blood flow. J Trauma. 2005;59:1521-31.

28. Greitz D, Wirestam R, Franck A, Nordell B, Thomsen C, Sthalberg F. Pulsatil brain movement and associated hydrondynamics studies by magnetix resonance phase imaging. The Monro-Kellie doctrine revisited. Neuroradiology. 1992;34:370-80.

29. Jokich PM, Rubin JM, Dohrmann GJ. Intraoperative ultrasonic evaluation of spinal cord motion. J Neurosurg. 1984;60:707-11.

30. Uftring SJ, Chu D, Alperin N, Levin DN. The mechanical state of intracranial tissues in elderly subjects studied by imaging CSF and brain pulsation. Magn Reson Imaging. 2000;18:991-6. 